\title{
A prospective study on inter-operator variability in semi-robotic software-based MRI/TRUS-fusion targeted prostate biopsies
}

\author{
Fabian Derigs ${ }^{1,4}\left(\right.$ C Samuel Doryumu ${ }^{1} \cdot$ Fabian Tollens $^{2} \cdot$ Dominik Nörenberg $^{2} \cdot$ Manuel Neuberger $^{1}$. \\ Jost von Hardenberg ${ }^{1} \cdot$ Maurice Stephan Michel ${ }^{1} \cdot$ Manuel Ritter $^{3} \cdot$ Niklas Westhoff $^{1}$
}

Received: 9 September 2021 / Accepted: 14 November 2021 / Published online: 26 November 2021

(c) The Author(s) 2021

\begin{abstract}
Purpose Magnetic resonance imaging (MRI)/ultrasound-fusion prostate biopsy (FB) comprises multiple steps each of which can cause alterations in targeted biopsy (TB) accuracy leading to false-negative results. The aim was to assess the interoperator variability of software-based fusion TB by targeting the same MRI-lesions by different urologists.

Methods In this prospective study, 142 patients eligible for analysis underwent software-based FB. TB of all lesions $(n=172)$ were carried out by two different urologists per patient ( $n=31$ urologists). We analyzed the number of mismatches [overall prostate cancer (PCa), clinically significant $\mathrm{PCa}$ (csPCa) and non-significant $\mathrm{PCa}(\mathrm{nsPCa})]$ between both performed TB per patient. In addition we evaluated factors contributing to inter-operator variability by uni- and multivariable analyses.

Results In $11.6 \%$ of all MRI-lesions (10.6\% of all patients) there was a mismatch between TB1 and TB2 in terms of overall prostate cancer (PCa detection. Regarding csPCa, patient-based mismatch occurred in $14.8 \%(n=21)$. Overall PCa and csPCa detection rate of TB1 and TB2 did not differ significantly on a per-patient and per-lesion level.

Analyses revealed a smaller lesion size as predictive for mismatches (OR 9.19, 95\% CI 2.02-41.83, $p<0.001$ ).

Conclusion Reproducibility and precision of targeting particularly small lesions is still limited although using software-based FB. Further improvements in image-fusion, segmentation, needle-guidance, and automatization are necessary.
\end{abstract}

Keywords Prostatic neoplasm · Multiparametric magnetic resonance imaging $\cdot$ Software-based fusion biopsy Reproducibility $\cdot$ Surgeon $\cdot$ Accuracy

Manuel Ritter and Niklas Westhoff: contributed equally.

Fabian Derigs

fabian.derigs@umm.de

1 Department of Urology and Urosurgery, Medical Faculty Mannheim, Heidelberg University, Mannheim, Germany

2 Department of Radiology and Nuclear Medicine, University Medical Center Mannheim, Medical Faculty Mannheim, Heidelberg University, Mannheim, Germany

3 Department of Urology and Pediatric Urology, University Hospital of Bonn, Bonn, Germany

4 Department of Urology and Urosurgery, University Medical Center Mannheim, Theodor-Kutzer-Ufer 1-3, 68167 Mannheim, Germany

\section{Introduction}

Multiparametric magnetic resonance imaging (mpMRI) in combination with targeted biopsy (TB) has greatly improved the identification of clinically significant prostate cancer (csPCa) [1]. Thus, MRI/ultrasound-fusion biopsy (FB) has been widely introduced in the last decade. Software-based image-fusion has gained greatest acceptance of fusion techniques [2].

Although TB has increased the detection rate for csPCa, yet a considerable amount of csPCa still remains undetected by TB [3-5]. This innovative approach of biopsy sampling represents a multi-step procedure involving different disciplines. Each step requires its own expertise which implies the occurrence of variations in the process. One of the preconditions for optimization of TB is the identification of its weaknesses to ensure no missing of csPCa.

The extent of inter-reader variability between radiologists and its implications for detection of prostate cancer (PCa) 
has already been described [6]. MRI result reporting from radiologists has also been shown to be of importance for biopsy performance [7]. Losses in accuracy in TB sampling have been analyzed in former research showing that experience of the urologist might also play an important role in cancer detection rates, CDRs $[8,9]$. However, when evaluating inter-operator variability in FB, most studies compared CDR of urologists with different levels of experience on different patients [8-10].

The aim of this prospective study was to assess the inter-operator variability and reproducibility of softwarebased fusion TB by targeting the same lesions by different urologists.

\section{Methods}

\section{Study design}

This prospective study was approved by the Local Institutional Ethical Review Board (approval no. 2015-403M-MA). All patients signed written informed consent for the intervention. Recruitment occurred at the University Medical Center Mannheim (Germany) between October 2016 and March 2021.

\section{Study population}

All men ( $\geq 18$ years of age) with (i) PCa suspicion [abnormal digital rectal examination, prostate specific antigen (PSA) elevation or abnormal MRI], (ii) persistent suspicion after one or more negative prior biopsies, or (iii) control biopsy while undergoing active surveillance, were eligible for inclusion.

\section{Acquisition and reporting of $\mathrm{mpMRI}$}

A mpMRI was performed in all patients either in the inhouse radiology department $(n=70)$ or external facilities $(n=65)$. For mpMRI acquisition, a magnetic field-strength of 3.0 T (Magnetom Skyra and Trio, Siemens Healthineers, Erlangen, Germany) was used at the in-house department and either $1.5 \mathrm{~T}$ or $3.0 \mathrm{~T}$ at external departments, mostly without use of an endorectal coil. T2-weighted sequences, diffusion-weighted imaging (DWI; b-values of 50, 400, $800 \mathrm{~s} / \mathrm{mm}^{2}$, additional $b$-value of $2000 \mathrm{~s} / \mathrm{mm}^{2}$ for Magnetom Skyra), and dynamic contrast enhanced perfusion sequences were obtained. Images were read and interpreted by respective uroradiologists who performed the mpMRI. In-house radiological appraisal was carried out or supervised by uroradiologists with more than 5 years of experience in urogenital imaging. MRI-lesions were scored according to latest PI-RADS guidelines (version dependent on the time of image acquisition).

\section{MRI/TRUS-fusion biopsy}

FB was performed under local or general anesthesia using the software-based robotic-assisted Artemis ${ }^{\mathrm{TM}}$ platform (Eigen, USA). Patients received either prophylactic or targeted antibiotic treatment dependent on preoperative rectal swap (and urine culture in case of risk factors for urinary tract infections). TB was performed independently by two urologists ( $n=31$ urologists) per patient. The first urologist contoured the prostate as well as suspicious lesion(s) within the MR images using the respective fusion software Profuse $^{\mathrm{TM}}$. Contouring was performed on the T2-weighted sequence as requested by taking also diffusion-weighted image and dynamic contrast-enhanced sequences into account. Afterwards, this urologist created a 3D-model of the prostate by the TRUS scan. After performing the TB of all lesions (TB1), the first urologist left the operating room, and the second urologist re-started the biopsy session with a new TRUS-scan and image-fusion procedure. TB sampling of the same lesions (TB2) was followed by the 12-core SB done by the second urologist.

\section{Data analysis}

Demographic, clinical, imaging and histopathological data were assessed by descriptive analysis. CDRs were analyzed on per-patient and per-lesion levels. An ISUP $\geq 2$ PCa was defined as clinically significant. Primary outcome was the number of mismatches [overall $\mathrm{PCa}$, csPCa and non-significant $\mathrm{PCa}$ (nsPCa)] between TBs of both urologists per patient. Secondary outcomes were factors that contribute to inter-operator variability in TB.

CDR were compared between biopsies using McNemar test. Cohen's $\kappa$ statistic was used for calculation of interoperator variability between the two urologists. Potential predictors for the occurrence of discrepancy between biopsy results were calculated by univariable analyses. Variables showing an odds ratio of $>1.5$ were further tested by multivariable analyses. For these calculations all lesions of PCa negative patients were excluded. For comparison of qualitative parameters Fisher's exact test was used. Experience as a factor for potential mismatches was evaluated by assessing the difference of the individual number of previously made in-house FB.

Analyses were performed using JMP ${ }^{\circledR} 15.0 .0$ and IBM $®$ SPSS $®$ Statistic Version 27 software. Level of statistical significance was set at $p<0.05$. 


\section{Results}

Characteristics of the study population are shown in Table 1. Overall, 155 patients received an MRI/TRUSfusion biopsy and signed the informed consent for the study. Patients who either had no complete study biopsy by a second urologist $(n=11)$ or received a control-biopsy after focal therapy $(n=2)$ were excluded.

The overall CDR was $71.8 \%$ (102/142 patients). SB found $64.8 \%$ PCa compared to $62.0 \%$ found by both TB $(\mathrm{TB} 1+\mathrm{TB} 2)(p=0.541)$. Total csPCa detection rate (csCDR) was $52.1 \%$ (41.5\% SB vs. $43.0 \% \mathrm{~TB}, p=0.851$ ). Out of 102 cancer positive biopsies on a per-patient level, $11 \mathrm{PCa}(10.8 \%)$ were detected exclusively by TB $(\mathrm{TB} 1+\mathrm{TB} 2)$ and $14 \mathrm{PCa}$ only by SB $(13.7 \%)(p=0.690)$. Out of 74 patients with a csPCa (SB + TB), 13 patients $(17.6 \%)$ had a csPCa finding solely in SB cores and 15 $(20.3 \%)$ only in TB cores (TB1+ TB2) $(p=0.851)$. On a per-lesion level, TB detection rates were 55.8\% (CDR) and $37.8 \%$ for csPCa.

There was no significant difference in CDR between TB1 and TB2 in all subgroups. The comparison of patientand lesion-based TB1 and TB2 detection rates is shown in Online Resource 1 . The lesion-based degree of agreement in detecting overall $\mathrm{PCa}(\kappa=0.56)$ and in detecting $\mathrm{nsPCa}$ $(\kappa=0.56)$ between TB1 and TB2 was by definition "moderate". Agreement in csPCa detection was by definition "substantial" ( $\kappa=0.65)$ (Online Resource 2$)$.

Figure 1 illustrates the number and types of mismatches between TB1 and TB2. In 20 MRI-suspicious lesions (11.6\%), corresponding to 15 patients $(10.6 \%)$, there was a mismatch between TB1 and TB2 in terms of overall cancer detection. Two out of 15 patients, whose lesions were only hit by one TB (TB1 or TB2), had a negative SB and no other positive lesions. In terms of csPCa detection, there was a patient-based mismatch of $14.8 \%(n=21)$ between TB1 and TB2. Six out of those 21 mismatch patients $(4.2 \%$ of total patients) had a benign or clinically insignificant finding (ISUP $=1$ ) in the SB.

Of all PCa positive lesions, in which both, TB1 and TB2, were cancer positive $(n=76)$, upgrading to a csPCa, either in TB1 or TB2, occurred in $13.2 \%(n=10)$.

The univariable and multivariable analyses of 112 lesions from the PCa positive patients for factors associated with mismatches revealed the size of the lesion $(\leq 12 \mathrm{~mm})$ described in the MRI as a predictive variable for mismatches $(p<0.001)$. Higher prostate volumes $(>41.21 \mathrm{ml})(\mathrm{OR} 2.15,95 \%$ CI $0.79-5.84, p=0.184)$ did not significantly correlate with mismatches (Online Resource 3).

Analyses of the $102 \mathrm{PCa}$ positive patients revealed that the size of the lesion was significantly smaller $(p=0.005)$
Table 1 Patient characteristics, results of mpMRI and MRI/TRUS fusion biopsy

\begin{tabular}{|c|c|}
\hline Variable & $n=142$ Patients \\
\hline \multicolumn{2}{|l|}{ Patient characteristics } \\
\hline Age, year (median, IQR) & $68.5(61-74)$ \\
\hline PSA, ng/ml (median, IQR) & $7.3(5.4-10.6)$ \\
\hline Prostate volume, $\mathrm{cm}^{3}$ (median, IQR) & $45.7(35.0-64.7)$ \\
\hline PSA-density, ng/ml ${ }^{2}$ (median, IQR) & $0.16(0.09-0.23)$ \\
\hline Suspicious digital rectal examination (\%) & $29(20.9)$ \\
\hline Biopsy naïve $(n, \%)$ & $91(64.1)$ \\
\hline Previous negative biopsy $(n, \%)$ & $44(31.0)$ \\
\hline Previous positive biopsy $(n, \%)$ & $7(4.9)$ \\
\hline \multicolumn{2}{|l|}{ Magnetic resonance imaging results } \\
\hline Unifocal lesion $(n, \%)$ & $115(81.0)$ \\
\hline Multifocal lesions $(n, \%)$ & $27(19.0)$ \\
\hline Index lesions $(n, \%)$ & 142 \\
\hline PI-RADS 5 & $40(28.2)$ \\
\hline PI-RADS 4 & $74(52.1)$ \\
\hline PI-RADS 3 & $27(19.0)$ \\
\hline PI-RADS 2 & $1(0.7)$ \\
\hline Total no. of lesions $(n, \%)$ & 172 \\
\hline PI-RADS 5 & $41(23.8)$ \\
\hline PI-RADS 4 & $86(50.0)$ \\
\hline PI-RADS 3 & $43(25.0)$ \\
\hline PI-RADS 2 & $2(1.2)$ \\
\hline $\begin{array}{l}\text { Max. diameter of index lesions, mm (median, } \\
\text { IQR) }\end{array}$ & $11(7.5-15.0)$ \\
\hline \multicolumn{2}{|l|}{ Biopsy results } \\
\hline SB cores per patient (median, IQR) & $12(12-12)$ \\
\hline \multicolumn{2}{|l|}{ Lesions with $(n, \%)$} \\
\hline $2 \mathrm{~TB}$ cores per operator & $136(79.1)$ \\
\hline $3 \mathrm{~TB}$ cores per operator & $31(18.0)$ \\
\hline $4 \mathrm{~TB}$ cores per operator & $5(2.9)$ \\
\hline TB positive per patient $(n, \%)$ & $88(62)$ \\
\hline SB positive per patient $(n, \%)$ & $92(64.8)$ \\
\hline TB positive per lesion $(n, \%)$ & $96(55.8)$ \\
\hline SB cores positive for PCa/total SB cores $(n, \%)$ & $284 / 1704(16.67)$ \\
\hline $\mathrm{TB}$ cores positive for $\mathrm{PCa} /$ total TB cores $(n, \%)$ & 286/688 (41.69) \\
\hline SB cancer core infiltration, mm (median, IQR) & $5.8(2.0-10.9)$ \\
\hline TB cancer core infiltration, mm (median, IQR) & $7.9(4.2-11.2)$ \\
\hline ISUP per patient ${ }^{\mathrm{a}}(n, \%)$ & 142 \\
\hline I & $28(19.7)$ \\
\hline II & $45(31.7)$ \\
\hline III & $14(9.9)$ \\
\hline IV & $9(6.3)$ \\
\hline $\mathrm{V}$ & $6(4.2)$ \\
\hline No cancer & $40(28.2)$ \\
\hline ISUP per lesion $^{\mathrm{b}}(n, \%)$ & 172 \\
\hline I & $31(18.0)$ \\
\hline II & $32(18.6)$ \\
\hline III & $8(4.7)$ \\
\hline IV & $19(11.0)$ \\
\hline
\end{tabular}


Table 1 (continued)

\begin{tabular}{ll}
\hline Variable & $n=142$ Patients \\
\hline VI & $6(3.5)$ \\
No cancer & $76(44.2)$ \\
Only TB positive of $(n, \%$ of PCa positive & $10(9.8)$ \\
$\quad$ patients) & \\
Only SB positive ( $n, \%$ of PCa positive patients) & $14(13.7)$ \\
Discrepancies in detecting PCa by TB $(n, \%$ & $15(14.7)$ \\
$\quad$ from all PCa positive patients) & \\
\hline
\end{tabular}

$P C a$ prostate cancer, PI-RADS Prostate Imaging Reporting and Data System, $T B$ targeted biopsy, $S B$ systematic biopsy

${ }^{a}$ By systematic biopsy and targeted biopsy

${ }^{\mathrm{b}}$ By targeted biopsy

and prostate volume was significantly bigger $(p=0.014)$ in the group of patients who were only cancer positive in $\mathrm{SB}(n=14)$.

Adverse events occurred in $7.0 \%(n=10)$ of patients. Hematuria was detected in $2.0 \%(n=3)$, urinary retention in $2.0 \%(n=3)$, rectal bleeding in $1.4 \%(n=2)$ and fever in $1.4 \%(n=2)$.

\section{Discussion}

Despite the superiority of FB compared to SB, many highrisk $\mathrm{PCa}$ still remains undetected by $\mathrm{TB}$ as shown by Ahdoot et al. [3]. They showed a misclassification rate of up to $13.6 \%$ of all csPCa bearing patients if SB would have been

Lesion based

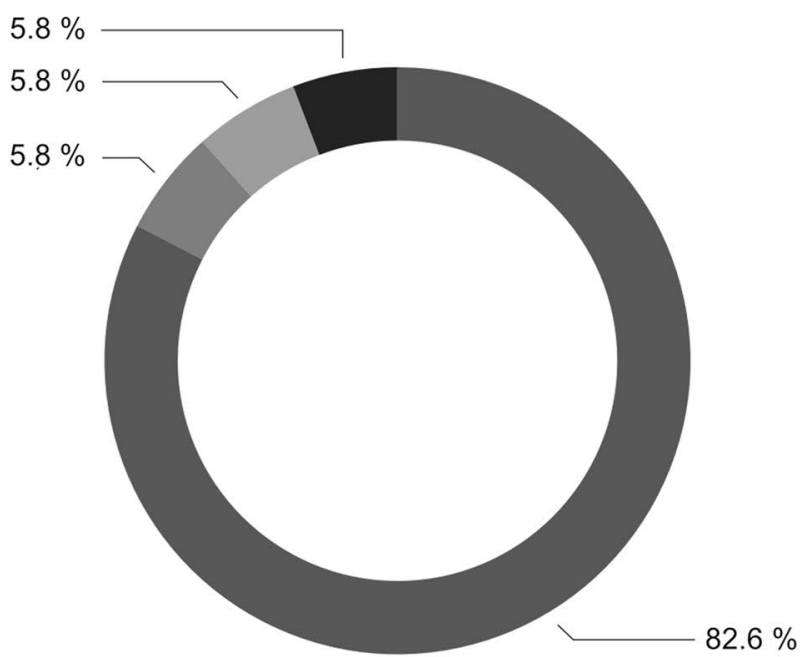

omitted [3]. In our study cohort, $17.6 \%$ of PCa patients with ISUP-score $\geq 2$ were misclassified by TB. Many factors have already been investigated which might influence FB [6-10]. These findings leave us the consideration of why the biopsy result of a suspicious lesion is negative.

The methodical approach of investigating inter-operator variability in the same patient by two different urologists to eliminate all confounding factors of procedure comparison has so far not been undertaken.

A key finding of our present study is that a considerable number of mismatches in PCa detection as well as in csPCa detection between both urologists could be observed. Although overall PCa and csPCa detection rate of TB1 and TB2 did not differ significantly on a per-patient and on a per-lesion level, a discrepancy in csPCa finding occurred in $14.8 \%$ of the study population. In total, a csPCa finding could have been missed in up to $4.2 \%$ of all patients, if the TB had been carried out only by one of the urologists. The remaining csPCa mismatch patients would have been covered by the SB, which accounts for $10.6 \%$, underlining the importance SB still has in this setting. This result also suggests that more than two cores should be taken from each target lesion in FB. Even though the number of TB cores was not a predictor for occurrence of mismatches here, several trials showed that up to $10 \%$ of patients would benefit from more than two cores per lesion [11].

Compared to Ahdoot et al., we identified a similar yield of PCa (62.0\% vs. $51.5 \%)$ and csPCa (43.0\% vs. $37.8 \%)$ with TB in our study [3]. Although detection rates between both TB did not differ significantly, the lesion-based level

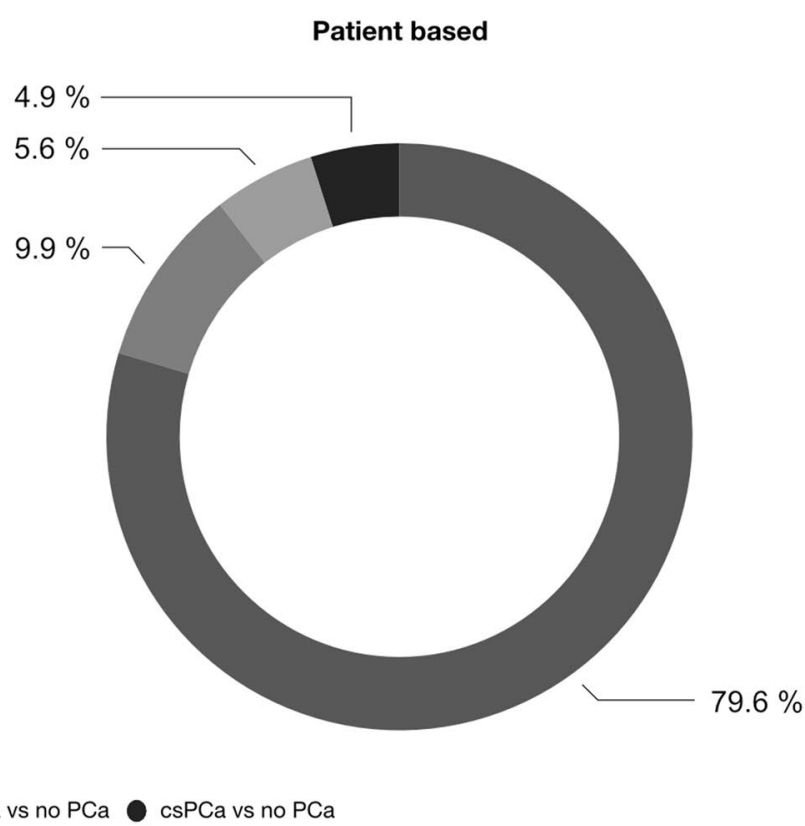

Fig. 1 Number and types of mismatches (discrepancies in histopathological biopsy results) between targeted biopsy 1 (TB1) and targeted biopsy 2 (TB2) 
of agreement was not optimal. In total, 20 lesion-based $\mathrm{PCa}$ detection discrepancies between TB1 and TB2 were found in our study. Half of those 20 lesions $(n=10)$ comprised a clinically significant cancer finding, emphasizing the imperfect reproducibility of $\mathrm{TB}$ even with the remarkable assistance of a biopsy platform.

In search of potential factors influencing the occurrence of mismatches between urologists, a smaller size of the MRIlesion was revealed as a significant predictor $(O R=9.19)$. The size of the lesion is also associated with the likelihood of both urologists missing the target, which supports the assumption that smaller PCa lesions are less likely to be identified by TB. This finding is in agreement with the recent study of Baco et al. describing a reduced csPCa detection rate of $50 \%$ for lesions $<0.5 \mathrm{ml}$ vs. $76 \%$ for lesions $>1 \mathrm{ml}$ in size $[12,13]$. It has been suggested that a perfect fusion of both images is necessary to reliably hit smaller targets. A small error in prostate and lesion boundary segmentation can already have a major impact on successful targeting [13]. The fact that both urologists in our study needed to carry out the delineation of the prostate in TRUS images, a procedure which requires high precision and is thus a source of targeting error, could explain these findings. The number of mismatches might be even higher if contouring of prostate and lesions in MRI-images was also done by each urologist separately. As discussed by Tay et al., ultrasoundsegmentation of the prostate necessitates a smooth and even sweep by the probe to avoid any displacements or rotation which may affect the shape of the 3D-construct, thus avoiding inaccurately displaying the target lesion. Sudden movements of the patients during the sweep, for example due to discomfort as well as prostate deformation by the application of too much pressure with the probe, can also alter the shape of the 3D-construct [13]. Although fusion platforms attempt to correct these alterations by using elastic registration algorithms and motion compensation, our results suggest the need for further optimization in this field [14]. Of particular importance, a deviation of the needle from the intended and predefined core path, for example due to its asymmetric bevel, is less likely to be compensated in smaller lesion sizes [13]. However, clinicians are partly able to adapt to this veering effect as they become more experienced over time and a (semi-)robotic needle guidance might further reduce the user-dependent effect. In contrast to previously published similar studies, we did not observe a large impact of the urologist's experience on cancer detection [8-10].

No significant correlation was shown between prostate volume and the occurrence of mismatches, which might be due to the rather small sample size of our cohort. However, inverse association of prostate volume with PCa detection by FB was previously described $[15,16]$. It is postulated that an increased prostate volume is associated with the deformation of the prostate during biopsy procedure leading to registration errors. Furthermore, the increased depth of the target lesion as may be found in an enlarged prostate is likely to be associated with increase in deviation of biopsy path [17].

The number of adverse events during and after the procedure are comparable to those in other studies [18].

Interpreting our results, a key limitation is the possibility of different surgical conditions for the first and second surgeon. It is suggested that the accuracy of hitting the target lesion on the real-time TRUS image during the second TB is decreased by tissue swelling caused by the first biopsy procedure. Regarding the discrepancies in csPCa finding, considerations to heterogeneity of tumor lesions should be made. Aihara et al. revealed in PCa specimens that with increased lesion size multiple grades of $\mathrm{PCa}$ can be present which are arranged in heterogeneous and unpredictable geographic interrelationships [19]. Therefore, evaluation of each surgeon's accuracy based on the grade of PCa might be limited. Despite the large number of different urologists taking biopsies, our results are still valuable since they reflect the real-world practice.

This study demonstrates that reproducibility and precision of targeting lesions suspicious for PCa is still limited, even with the high-level support of a semi-robotically software-based fusion biopsy platform. Although the detection rate of $\mathrm{PCa}$ and csPCa can be markedly improved by FB, discrepancies in biopsy results between individual urologists can still be observed. This insight should serve as an incentive for further improvements in image fusion, segmentation, needle guidance as well as automatization of the procedure so that even unexperienced clinicians are able to reliably hit a small lesion in an enlarged prostate.

Supplementary Information The online version contains supplementary material available at https://doi.org/10.1007/s00345-021-03891-3.

Author contributions NW: project development, manuscript writing and data analysis; MR: project development, manuscript editing; FD: data collection, data analysis and manuscript writing; SD: data collection, data analysis, and manuscript editing; FT: data collection and manuscript editing; MN: manuscript editing; MSM: manuscript editing; JvH: manuscript editing and DN: manuscript editing.

Funding Open Access funding enabled and organized by Projekt DEAL. No funding was received for conducting this study.

Data availability The raw data supporting the conclusions of this article will be made available by the authors, without undue reservations.Consent to publish Patients signed informed consent regarding publishing their data. 


\section{Declarations}

Conflict of interest All authors declare no potential conflicts of interest.

Ethical approval This prospective study was approved by the Institutional Ethical Review Board (2015-403M-MA).

Informed consent Informed consent was obtained from all individual participants included in the study.

Open Access This article is licensed under a Creative Commons Attribution 4.0 International License, which permits use, sharing, adaptation, distribution and reproduction in any medium or format, as long as you give appropriate credit to the original author(s) and the source, provide a link to the Creative Commons licence, and indicate if changes were made. The images or other third party material in this article are included in the article's Creative Commons licence, unless indicated otherwise in a credit line to the material. If material is not included in the article's Creative Commons licence and your intended use is not permitted by statutory regulation or exceeds the permitted use, you will need to obtain permission directly from the copyright holder. To view a copy of this licence, visit http://creativecommons.org/licenses/by/4.0/.

\section{References}

1. Drost FH, Osses DF, Nieboer D, Steyerberg EW, Bangma CH, Roobol MJ, Schoots IG (2019) Prostate MRI, with or without MRI-targeted biopsy, and systematic biopsy for detecting prostate cancer. Cochrane Database Syst Rev 4:CD012663. https://doi.org/ 10.1002/14651858.CD012663.pub2

2. van Luijtelaar A, Bomers J, Futterer J (2019) A comparison of magnetic resonance imaging techniques used to secure biopsies in prostate cancer patients. Expert Rev Anticancer Ther 19(8):705716. https://doi.org/10.1080/14737140.2019.1641086

3. Ahdoot M, Wilbur AR, Reese SE, Lebastchi AH, Mehralivand S, Gomella PT, Bloom J, Gurram S, Siddiqui M, Pinsky P, Parnes H, Linehan WM, Merino M, Choyke PL, Shih JH, Turkbey B, Wood BJ, Pinto PA (2020) MRI-targeted, systematic, and combined biopsy for prostate cancer diagnosis. N Engl J Med 382(10):917928. https://doi.org/10.1056/NEJMoa1910038

4. Mannaerts CK, Kajtazovic A, Lodeizen OAP, Gayet M, Engelbrecht MRW, Jager GJ, Wijkstra H, de Reijke TM, Beerlage HP (2019) The added value of systematic biopsy in men with suspicion of prostate cancer undergoing multiparametric MRI-targeted biopsy. Urol Oncol 37(5):298.e291-298.e299. https://doi.org/10. 1016/j.urolonc.2019.01.005

5. Rouviere O, Puech P, Renard-Penna R, Claudon M, Roy C, MegeLechevallier F, Decaussin-Petrucci M, Dubreuil-Chambardel M, Magaud L, Remontet L, Ruffion A, Colombel M, Crouzet S, Schott AM, Lemaitre L, Rabilloud M, Grenier N, M-F Investigators (2019) Use of prostate systematic and targeted biopsy on the basis of multiparametric MRI in biopsy-naive patients (MRIFIRST): a prospective, multicentre, paired diagnostic study. Lancet Oncol 20(1):100-109. https://doi.org/10.1016/S1470-2045(18) 30569-2

6. Smith CP, Harmon SA, Barrett T, Bittencourt LK, Law YM, Shebel H, An JY, Czarniecki M, Mehralivand S, Coskun M, Wood BJ, Pinto PA, Shih JH, Choyke PL, Turkbey B (2019) Intra- and interreader reproducibility of PI-RADSv2: a multireader study. J Magn Reson Imaging 49(6):1694-1703. https://doi.org/10.1002/ jmri.26555
7. Westhoff N, Siegel F, Peter C, Hetjens S, Porubsky S, Martini T, von Hardenberg J, Michel MS, Budjan J, Ritter M (2019) Defining the target prior to prostate fusion biopsy: the effect of MRI reporting on cancer detection. World J Urol 37(2):327-335. https://doi. org/10.1007/s00345-018-2400-x

8. Calio B, Sidana A, Sugano D, Gaur S, Jain A, Maruf M, Xu S, Yan P, Kruecker J, Merino M, Choyke P, Turkbey B, Wood B, Pinto P (2017) Changes in prostate cancer detection rate of MRI-TRUS fusion vs systematic biopsy over time: evidence of a learning curve. Prostate Cancer Prostatic Dis 20(4):436-441. https://doi. org/10.1038/pcan.2017.34

9. Mager R, Brandt MP, Borgmann H, Gust KM, Haferkamp A, Kurosch M (2017) From novice to expert: analyzing the learning curve for MRI-transrectal ultrasonography fusion-guided transrectal prostate biopsy. Int Urol Nephrol 49(9):1537-1544. https://doi. org/10.1007/s11255-017-1642-7

10. Zalesky M, Stejskal J, Minarik I, Adamcova V, Babjuk M, Zachoval R (2020) Cancer detection rates and inter-examiner variability of MRI/TRUS fusion targeted biopsy and systematic transrectal biopsy. Biomed Pap Med Fac Univ Palacky Olomouc Czech Repub 164(3):314-319. https://doi.org/10.5507/bp.2019.050

11. Kenigsberg AP, Renson A, Rosenkrantz AB, Huang R, Wysock JS, Taneja SS, Bjurlin MA (2018) Optimizing the number of cores targeted during prostate magnetic resonance imaging fusion target biopsy. Eur Urol Oncol 1(5):418-425. https://doi.org/10.1016/j. euo.2018.09.006

12. Baco E, Rud E, Ukimura O, Vlatkovic L, Svindland A, Matsugasumi T, Bernhard JC, Rewcastle JC, Eggesbo HB (2014) Effect of targeted biopsy guided by elastic image fusion of MRI with 3D-TRUS on diagnosis of anterior prostate cancer. Urol Oncol 32(8):1300-1307. https://doi.org/10.1016/j.urolonc.2014.07.014

13. Tay KJ, Gupta RT, Rastinehad AR, Tsivian E, Freedland SJ, Moul JW, Polascik TJ (2016) Navigating MRI-TRUS fusion biopsy: optimizing the process and avoiding technical pitfalls. Expert Rev Anticancer Ther 16(3):303-311. https://doi.org/10.1586/14737 140.2016.1131155

14. Gold SA, Hale GR, Bloom JB, Smith CP, Rayn KN, Valera V, Wood BJ, Choyke PL, Turkbey B, Pinto PA (2019) Follow-up of negative MRI-targeted prostate biopsies: when are we missing cancer? World J Urol 37(2):235-241. https://doi.org/10.1007/ s00345-018-2337-0

15. Muthigi A, George AK, Sidana A, Kongnyuy M, Simon R, Moreno V, Merino MJ, Choyke PL, Turkbey B, Wood BJ, Pinto PA (2017) Missing the mark: prostate cancer upgrading by systematic biopsy over magnetic resonance imaging/transrectal ultrasound fusion biopsy. J Urol 197(2):327-334. https://doi.org/10. 1016/j.juro.2016.08.097

16. Walton Diaz A, Hoang AN, Turkbey B, Hong CW, Truong H, Sterling T, Rais-Bahrami S, Siddiqui MM, Stamatakis L, Vourganti S, Nix J, Logan J, Harris C, Weintraub M, Chua C, Merino MJ, Choyke P, Wood BJ, Pinto PA (2013) Can magnetic resonance-ultrasound fusion biopsy improve cancer detection in enlarged prostates? J Urol 190(6):2020-2025. https://doi.org/10. 1016/j.juro.2013.05.118

17. Wooten III WJ, Nye JA, Schuster DM, Nieh PT, Master VA, Votaw JR, Fei B (2013) Accuracy evaluation of a 3D ultrasoundguided biopsy system. In: Proceedings of the SPIE International Society for Optical Engineering, vol 8671. https://doi.org/10. $1117 / 12.2007695$

18. Borghesi M, Ahmed H, Nam R, Schaeffer E, Schiavina R, Taneja S, Weidner W, Loeb S (2017) Complications after systematic, random, and image-guided prostate biopsy. Eur Urol 71(3):353-365. https://doi.org/10.1016/j.eururo.2016.08.004

19. Aihara M, Wheeler TM, Ohori M, Scardino PT (1994) Heterogeneity of prostate cancer in radical prostatectomy specimens. 
Urology 43(1):60-66; discussion 66-67. doi: https://doi.org/10. 1016/s0090-4295(94)80264-5

Publisher's Note Springer Nature remains neutral with regard to jurisdictional claims in published maps and institutional affiliations. 